$20 \begin{aligned} & \text { Pesquisa Florestal Brasileira } \\ & \text { Brazilian Journal of Foresty Research } \\ & \text { www.cnpf.embrapa.br/pfb }\end{aligned}$

\title{
Dendroecologia de Cedrela fissilis na Floresta Ombrófila Mista
}

\author{
Andrea Chizzotti Cusatis ${ }^{1}$, Paulo André Trazzi ${ }^{1}$, Mário Dobner $\mathrm{Jr}^{1}$, Antonio Rioyei Higa ${ }^{1}$ \\ ${ }^{1}$ Universidade Federal do Paraná, Departamento de Engenharia Florestal, Av. Pref. Lothário Meissner, 900, CEP 80210-170, Curitiba, PR, Brasil
}

\author{
"Autor correspondente: \\ acusatis@hotmail.com \\ Termos para indexação: \\ Cedro-rosa \\ Dendrocronologia \\ Meliaceae \\ Silvicultura

\section{Index terms:} \\ Tropical cedar \\ Dendrocronology \\ Meliaceae \\ Forestry
}

Histórico do artigo:

Recebido em 17/01/2013

Aprovado em 19/09/2013

Publicado em 30/09/2013

doi: $10.4336 / 2013 . p f b .33 .75 .474$
Resumo - Embora a madeira de Cedrela fissilis Vell. seja de grande interesse comercial, seu uso é limitado em função da escassez de informações quanto à silvicultura da espécie. O objetivo deste trabalho foi avaliar o crescimento e a correlação deste com variáveis climáticas em seis municípios na divisa dos estados do Paraná e Santa Catarina. Amostras radiais do lenho foram coletadas de 42 indivíduos. Após secas e lixadas, os anéis de crescimento foram marcados, mensurados e avaliados. O incremento médio em diâmetro foi de $1,1 \mathrm{~cm}^{2} \mathrm{ano}^{-1}$. Considerando um diâmetro de corte de $40 \mathrm{~cm}$, estimam-se rotações de 30 a 36 anos para a espécie. De 42 indivíduos amostrados foi possível codatar 28 séries de crescimento, apresentando uma intercorrelação de 0,551 . A correlação de Pearson entre as variáveis climáticas e o crescimento radial $C$. fissilis não apresentou resultados significativos para a maioria das variáveis. Contudo, a umidade relativa do ar em dezembro foi significativa e inversamente proporcional ao crescimento radial.

\section{Dendroecology of Cedrela fissilis from Araucaria Forest}

\begin{abstract}
Although the wood from Cedrela fissilis Vell. has great commercial interest, its use is limited due to the lack of information regarding forestry of the species. The aim of this study was to evaluate growth and its correlations with climate variables in six districts at the eastern border of Paraná and Santa Catarina states. Radial wood samples were collected from 42 individuals. Once dried and sanded the annual growth rings were marked, measured and evaluated. The average diameter increment was $1.1 \mathrm{~cm}$ year ${ }^{-1}$. Considering a cutting diameter of $40 \mathrm{~cm}$ it was estimated rotations of 30 to 36 years to the species. From the 42 individuals sampled, it was possible to cross date 28 growth series, which presented an inter-correlation of 0.551 . Pearson correlation analysis between radial growth and climate data did not show significant values for most of the variables. However, the relative humidity of the air in December was significant and inversely proportional to the radial growth.
\end{abstract}

\section{Introdução}

Cedrela fissilis ocorre naturalmente do Rio Grande do Sul até Minas Gerais, nas Florestas Estacionais Semidecíduas, Ombrófila Mista e, em menor expressão, na Ombrófila Densa da costa atlântica. A espécie tem preferência por solos úmidos, como os encontrados nos vales e planícies aluviais (Reitz, 1984).

O potencial madeireiro de C. fissilis é conferido pela excelente estabilidade dimensional, bem como a coloração, variando do bege rosado ao castanho avermelhado (Reitz et al., 1978). O alto valor da madeira serrada, estimado em mais de R $\$ 2.500 \mathrm{o} \mathrm{m}^{-3}$ (São Paulo, 2008), deve-se ao seu uso nobre, como instrumentos musicais, carpintaria, marcenaria, decoração de interiores, construção naval e aeronáutica, entre outros (Rizzini, 1978).

Informações sobre a ecologia e o crescimento de espécies nativas possuem grande importância no estabelecimento de critérios para a silvicultura das 
mesmas, uma vez que esses dados, quando disponíveis, são utilizados para inferir sobre produção, produtividade e idade de corte, quando se cogita a exploração comercial de uma espécie.

O conhecimento da dinâmica de crescimento das árvores e dos fatores climáticos como causas de variação das propriedades da madeira são, respectivamente, atribuídos aos pesquisadores Harold C. Fritts e Fritz H. Schweingruber, como contribuições pioneiras da dendrocronologia à silvicultura (Downes et al., 2002).

A análise da influência dos fatores ambientais no crescimento anual das árvores tem como premissa o 'princípio do fator limitante' (Grissino-Mayer, 1996; Tomazello Filho et al., 2001). De acordo com este princípio, o crescimento de uma árvore é sempre controlado por determinados fatores externos (água, luz, temperatura, etc.) e demais fatores internos (genótipo, fitohormônios, etc.).

Schweingruber (2007) relata que condições ambientais extremas, tais como secas e períodos de frio, limitam o crescimento dos anéis de crescimento, permitindo, assim, o estudo do efeito das variações ambientais no crescimento do tronco das árvores, também conhecido como dendroecologia.

Diversos trabalhos realizados nas regiões Sul e Sudeste do Brasil, comprovam a formação de anéis anuais de crescimento e o potencial dendrocronológico de diversas espécies (Seitz, 1988; Seitz \& Kaninnen, 1989; Tomazello Filho et al., 2004; Iwasaki-Marochi, 2007; Medeiros et al., 2008; Mattos et al., 2010; Rauber, 2010; Oliveira et al., 2010, 2011; Cardoso, 2012).

Segundo Tomazello Filho et al. (2000), Marcati et al. (2006) e Iwasaki-Marochi (2007), C. fissilis é uma espécie indicada para estudos dendrocronológicos, uma vez que seus anéis de crescimento são evidentes e com formação anual. Os anéis formam camadas demarcadas por faixas de parênquima axial marginal e anéis semiporosos, tornando possíveis: a detecção dos limites dos anéis de crescimento, a estimativa de sua idade e a medição do crescimento diamétrico anual. As faixas marginais são constituídas de células de parênquima estabelecidas no final de um período de atividade cambial e no início da seguinte. O período de dormência cambial está correlacionado com a estação de seca, no caso da formação Estacional Semidecidual, e a queda das folhas, já o período de atividade cambial está relacionado à estação chuvosa e à presença de folhas maduras (Marcati et al., 2006).
A compreensão de como uma população de determinada espécie florestal responde às variáveis meteorológicas é importante para decisões relacionadas à silvicultura e para a análise de possíveis efeitos de mudanças climáticas no crescimento das mesmas. Ainda, o estabelecimento de um modelo de crescimento para uma espécie em domesticação pode contribuir para estimativas da viabilidade econômica para determinada região.

O objetivo do presente trabalho foi estabelecer o incremento em diâmetro, avaliar as correlações das séries temporais da largura dos anéis de crescimento, construir uma cronologia para a espécie na área de estudo e testar a correlação da cronologia mestra com variáveis meteorológicas.

\section{Material e métodos}

Para a descrição do local e análise das correlações entre o clima e o crescimento das árvores foram utilizados todos os dados meteorológicos disponíveis da área de amostragem (Tabela 1). Valores médios mensais para os municípios de Rio Negrinho, São Bento do Sul e Lapa, durante o período 1970 a 2010, são apresentados na Figura 1.

Para a realização das análises de correlação, foram empregados valores gerados pelo aplicativo MET, integrante da livraria de programas dendrocronológicos (DPL), o qual gera uma série única a partir de dados meteorológicos de estações distintas. $\mathrm{O}$ aplicativo estima valores de dados mensais a partir do cálculo da média mensal da estação assim como da média de outras estações para aquele mês e ano. $\mathrm{O}$ desvio padrão do mês daquela estação é multiplicado pela média regional do mês e ano, e o valor médio do mês daquela estação é adicionado. Assim, novos valores são estimados para aquelas estações com dados ausentes (Holmes, 1994).

Foram coletadas amostras radiais não destrutivas com 1,2 cm de diâmetro do lenho de 42 indivíduos em fevereiro de 2012, distribuídos em seis municípios na divisa entre Paraná e Santa Catarina.

A altura de coleta das baguetas foi de $1,3 \mathrm{~m}$ acima do nível do solo (DAP). A medição do DAP foi feita com fita dendrométrica e a altura total e comercial foram determinadas com hipsômetro Suunto ${ }^{\circledR}$. Do total de amostras coletadas, 28 baguetas foram consideradas viáveis para análise, considerando a integridade do lenho e a boa correlação com a série de crescimento, embora nem todas as medulas tenham sido atingidas em função da limitação do comprimento do trado $(30 \mathrm{~cm})$. A localização das 28 árvores utilizadas nas análises foram apresentadas na Figura 2. 
Tabela 1. Dados meteorológicos por município, períodos analisados e fontes.

\begin{tabular}{|c|c|c|c|}
\hline Dado & Município & Período & Fonte \\
\hline Precipitação & São Bento do Sul & 1970 a 1984 & $\mathrm{EPAGRI}^{(1)} / \mathrm{ANA}^{(2)}$ \\
\hline Precipitação & Rio Negrinho & 1976 a 2011 & EPAGRI/ANA \\
\hline Precipitação & Rio Negrinho & 1990 a 2011 & EPAGRI \\
\hline Precipitação & Lapa & 1988 a 2011 & IAPAR $^{(3)}$ \\
\hline Temp..$^{(4)}$ média & Rio Negrinho & 1990 a 2011 & EPAGRI \\
\hline Temp. média & Lapa & 1988 a 2011 & IAPAR \\
\hline Temp. média & Lapa & 1997 a 2011 & SIMEPAR $^{(5)}$ \\
\hline Temp. mínima absoluta & Rio Negrinho & 1990 a 2011 & EPAGRI \\
\hline Temp. mínima absoluta & Lapa & 1997 a 2011 & SIMEPAR \\
\hline Temp. mínima média & Lapa & 1988 a 2011 & IAPAR \\
\hline Temp. máxima absoluta & Rio Negrinho & 1990 a 2011 & EPAGRI \\
\hline Temp. máxima absoluta & Lapa & 1997 a 2011 & SIMEPAR \\
\hline Temp. máxima média & Lapa & 1988 a 2011 & IAPAR \\
\hline Umidade relativa & Rio Negrinho & 1990 a 2011 & EPAGRI \\
\hline Umidade relativa & Lapa & 1988 a 2011 & IAPAR \\
\hline Dias de chuva mês ${ }^{-1}$ & São Bento do Sul & 1970 a 1984 & EPAGRI/ANA \\
\hline Dias de chuva mês ${ }^{-1}$ & Rio Negrinho & 1976 a 2011 & EPAGRI/ANA \\
\hline Dias de chuva mês ${ }^{-1}$ & Rio Negrinho & 1990 a 2011 & EPAGRI \\
\hline Geadas mês ${ }^{-1}$ & Lapa & 1989 a 2011 & SIMEPAR \\
\hline Geadas mês $^{-1}$ & Lapa & 1990 a 2011 & IAPAR \\
\hline
\end{tabular}

${ }^{(1)}$ Empresa de Pesquisa Agropecuária e Extensão Rural de Santa Catarina. ${ }^{(2)}$ Agência Nacional de Águas. ${ }^{(3)}$ Instituto Agronômico do Paraná. ${ }^{(4)}$ Temperatura. ${ }^{(5)}$ Sistema Meteorológico do Paraná.

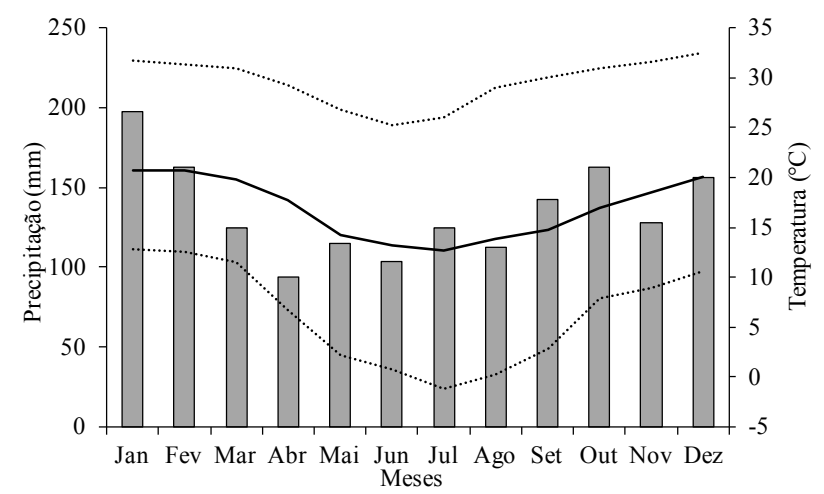

Figura 1.Precipitação (colunas), temperatura média (linha), temperaturas mínima e máxima (linhas pontilhadas): valores médios mensais dos municípios São Bento do Sul, Rio Negrinho e Campo Alegre, durante o período analisado (19702011).

Após secas, as baguetas foram lixadas progressivamente com lixas de granulometria de 60, 80, 120 e 200 com o objetivo de tornar os anéis de crescimento mais evidentes. Estas foram então digitalizadas em scanner (HP ScanJet $5100 \mathrm{C}, 600 \mathrm{dpi}$ ), as imagens foram calibradas com um escalímetro e as larguras dos anéis foram medidas com uma acurácia de $0,1 \mathrm{~mm}$, utilizando o software Image Pro Plus, versão 4.5.

Medir a espessura dos anéis anuais de crescimento permitiu determinar o DAP dos indivíduos em diferentes idades. A partir dos 345 pares de dados DAP x idade, foi ajustado o modelo de crescimento Chapman-Richards (Pretzsch, 2009), o qual estima o diâmetro a partir da variável independente idade em anos.

A caracterização do ambiente no entorno das árvores amostradas está compilada na Tabela 2.

Em função do período de crescimento no hemisfério sul ser da primavera do ano $t$ ao outono do ano $t+1$, abrangendo dois anos do calendário, as análises de correlação consideraram o período de formação do anel.

A codatação das sequências de crescimento foi verificada com o auxílio do software Cofecha (Holmes, 1996; Grissino-Mayer, 1997), selecionando no controle de opções segmentos com 30 anos com deslocamento de 15 anos. Tal comprimento de segmento de análise possui correlação crítica de $0,423(p<0,01)$. 


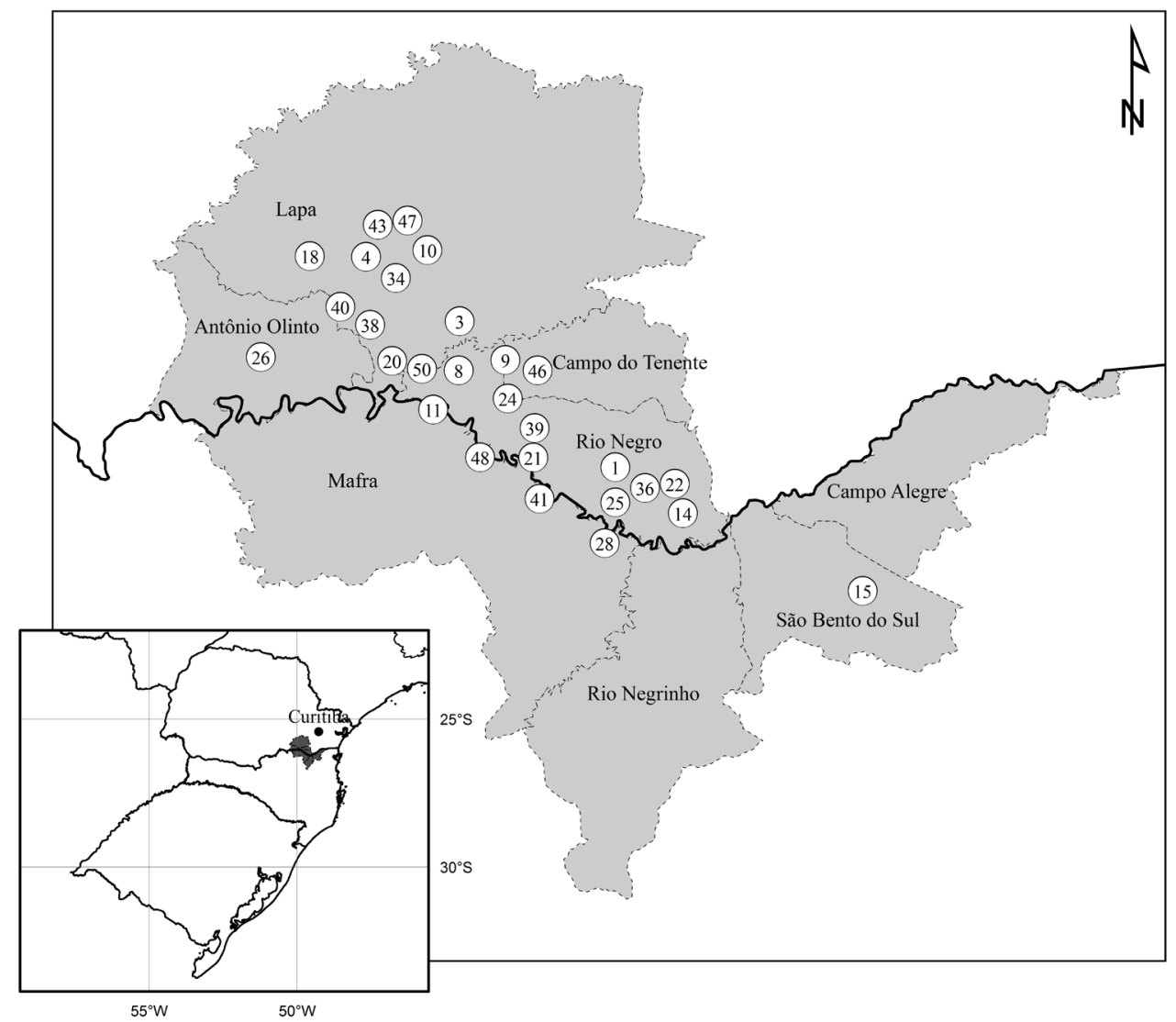

Figura 2.Localização geográfica das matrizes analisadas; destaque para os estados do Sul do Brasil.

Embora um indivíduo analisado tenha apresentado anéis de crescimento desde 1971, somente a partir de 1974 houve duas ou mais séries de crescimento disponíveis. As séries de crescimento foram padronizadas com o auxílio do software Arstan, desenvolvido para gerar cronologias mestres, removendo a influência da idade das árvores e as alterações no crescimento em função da dinâmica florestal (Copenheaver et al., 2011). A cronologia mestre gerada possui valores $>1$ ou $<1$, significando períodos de crescimento acima ou abaixo da função de crescimento aplicada para estandartização dos dados.

Foi testada a correlação da cronologia mestre com os dados meteorológicos mensais de temperatura (mínima, média e máxima), precipitação acumulada, umidade relativa média, número de dias com chuva e número de dias com geada. As correlações entre séries dendrocronológicas e os dados meteorológicos foram investigadas com a aplicação de análise de correlação de Pearson.

Estabeleceu-se que o último mês de crescimento foi agosto, considerando os dados meteorológicos dos 12 meses anteriores ao término do período vegetativo (setembro). Utilizou-se como referência os resultados obtidos por Marcati et al. (2006), que estudaram Cedrela fissilis no sudeste do Brasil e verificaram que atividade cambial corresponde a estação chuvosa. 
Tabela 2. Correlação da cronologia individual com as outras séries de anéis de crescimento, DAP, altura total, ponto de inversão morfológica (PIM), município e caracterização do entorno das árvores de Cedrela fissilis amostradas.

\begin{tabular}{|c|c|c|c|c|c|c|}
\hline Árvore & Correlação & $\begin{array}{l}\text { DAP } \\
(\mathbf{c m})\end{array}$ & $\begin{array}{l}\text { Altura } \\
(\mathbf{m})\end{array}$ & $\underset{(m)}{\text { PIM }}$ & Município & Caracterização \\
\hline 1 & 0,541 & 57 & 16 & 3,5 & Rio Negro, PR & $\begin{array}{l}\text { À margem de estrada, entre fragmento de floresta nativa e } \\
\text { plantio de eucalipto, bifurcada. }\end{array}$ \\
\hline 3 & 0,651 & 46,3 & 17,5 & 10 & Lapa, PR & Isolada. \\
\hline 4 & 0,569 & 56 & 17 & 2,8 & Lapa, PR & À margem de estrada, presença de lianas. \\
\hline 8 & 0,640 & 40,7 & 14,5 & 6,5 & Rio Negro, PR & Em fragmento alterado por pastoreio de gado. \\
\hline 9 & 0,424 & 24,3 & 12,5 & 4,5 & Campo do Tenente, PR & À margem de estrada e plantio de pínus. \\
\hline 10 & 0,425 & 48,8 & 19 & 6,5 & Lapa, PR & Em pomar frutífero com gado e próximo a acesso de estrada. \\
\hline 11 & 0,638 & 58,5 & 14,5 & 3 & Rio Negro, $\mathrm{PR}$ & $\begin{array}{l}\text { À margem de estrada e fragmento de floresta nativa muito } \\
\text { alterada. }\end{array}$ \\
\hline 14 & 0,553 & 34,3 & 22 & 10 & Rio Negro, PR & Em fragmento de floresta nativa alterada. \\
\hline 15 & 0,282 & 41,2 & 22 & 7 & São Bento do Sul, SC & $\begin{array}{l}\text { À margem de estrada, em fragmento de floresta nativa alterada, } \\
\text { terreno forte ondulado. }\end{array}$ \\
\hline 18 & 0,783 & 37,1 & 12 & 5,8 & Lapa, PR & $\begin{array}{l}\text { À margem de rodovia, área de floresta nativa em regeneração; } \\
\text { bifurcada a } 1,3 \mathrm{~m} \text {. }\end{array}$ \\
\hline 20 & 0,463 & 82,9 & 17,5 & 3 & Lapa, PR & À margem de estrada, em fragmento de floresta nativa. \\
\hline 22 & 0,642 & 44,7 & 19 & 6 & Rio Negro, PR & $\begin{array}{l}\text { À margem de plantio de soja e regeneração natural de floresta } \\
\text { nativa. }\end{array}$ \\
\hline 24 & 0,528 & 64,5 & 16,5 & 4 & Rio Negro, PR & $\begin{array}{l}\text { Em área alterada, bambus no entorno, próximo a estrada de } \\
\text { terra. }\end{array}$ \\
\hline 25 & 0,674 & 44 & 12 & 2,8 & Mafra, SC & Isolada. \\
\hline 26 & 0,543 & 63 & 20 & 9 & Antônio Olinto, PR & Plantio de pínus e fragmento de floresta nativa no entorno. \\
\hline 28 & 0,613 & 39,5 & 18 & 4,6 & Rio Negro, PR & À margem de estrada, em fragmento de floresta nativa. \\
\hline 34 & 0,621 & 59 & 16 & 4 & Lapa, PR & Isolada. \\
\hline 36 & 0,519 & 32,8 & 14 & 7,5 & Rio Negro, PR & Em fragmento de floresta nativa. \\
\hline 37 & 0,717 & 53 & 12 & 1,5 & Rio Negro, PR & $\begin{array}{l}\text { À margem de estrada, entre eucaliptos; cicatriz no tronco, } \\
\text { vestígios de ataque de Hypsipyla. grandella. }\end{array}$ \\
\hline 38 & 0,376 & 54 & 16 & 3,5 & Lapa, PR & À margem de fragmento de floresta nativa. \\
\hline 39 & 0,729 & 40,9 & 18,5 & 5,5 & Rio Negro, PR & Em fragmento de floresta nativa alterada. \\
\hline 40 & 0,449 & 32,7 & 11 & 7,5 & Lapa, PR & $\begin{array}{l}\text { À margem de rodovia em fragmento de floresta nativa, árvore } \\
\text { bifurcada a } 1,4 \mathrm{~m} \text {. }\end{array}$ \\
\hline 41 & 0,298 & 32 & 17 & 8 & Mafra, SC & À margem da rodovia em fragmento de floresta nativa. \\
\hline 43 & 0,551 & 63,8 & 19,5 & 3,5 & Lapa, PR & $\begin{array}{l}\text { À margem da rodovia, em fragmento de floresta nativa em } \\
\text { regeneração, presença de taquara. }\end{array}$ \\
\hline 46 & 0,705 & 41,8 & 3 & - & Rio Negro, PR & $\begin{array}{l}\text { Em fragmento de floresta nativa alterada, árvore quebrada, } \\
\text { lianas em abundância. }\end{array}$ \\
\hline 47 & 0,294 & 84,8 & 22 & 4 & Lapa, PR & $\begin{array}{l}\text { À margem da rodovia, em fragmento de floresta nativa em } \\
\text { regeneração. }\end{array}$ \\
\hline 48 & 0,615 & 38,5 & 17 & 5,5 & Campo do Tenente, PR & À margem de estrada. \\
\hline 50 & 0,620 & - & - & - & Lapa, PR & $\begin{array}{l}\text { Isolada entre plantio de pinus em sistema silvipastoril e } \\
\text { cruzamento de estrada de terra. }\end{array}$ \\
\hline
\end{tabular}




\section{Resultados e discussão}

Dentre as 42 árvores amostradas, três indivíduos apresentaram lenho danificado, decorrente da ação de fungos, impossibilitando a medição dos seus anéis de crescimento em, pelo menos, parte da amostra. Das 39 árvores medidas, 11 apresentaram série temporal de crescimento pouco ou negativamente correlacionado à série de crescimento média das demais (Tabela 2). Além disso, a remoção da amostra do lenho com trado de incremento foi capaz de produzir um raio completo (casca - medula) em apenas 11 indivíduos. Por este motivo, foram estas as árvores utilizadas para a análise gráfica do crescimento, apresentada na Figura 3.

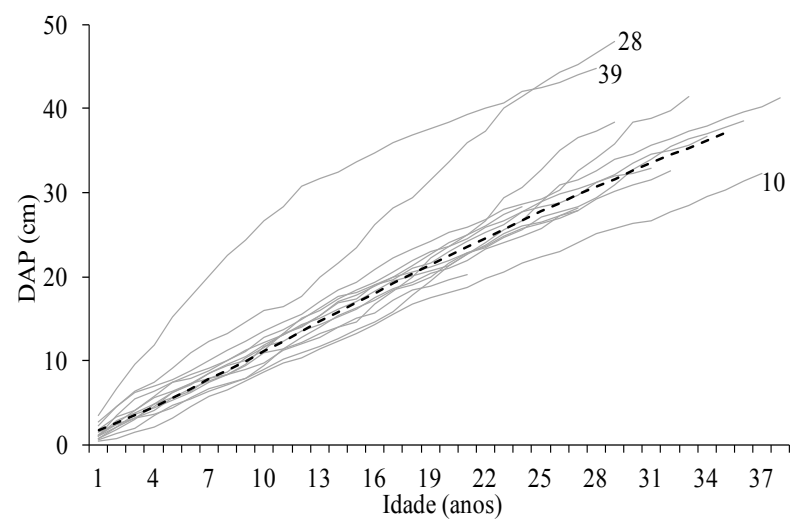

Figura 3. Crescimento diamétrico acumulado das árvores de C. fissilis; destaque para o padrão de crescimento diferente do conjunto para os indivíduos 10, 28 e 39; modelo ChapmanRichards de crescimento (linha tracejada) para todas as árvores, com exceção das destacadas.

Apesar de Iwasaki-Marochi (2007) relatar facilidade na distinção dos anéis anuais de crescimento, o uso do software Cofecha foi necessário para a identificação de falsos anéis, mais difíceis de detectar durante a marcação por se tratarem de baguetas e não discos transversais. Segmentos da série de crescimento com indicações consistentes de ausência de anel ou com falso anel foram confirmadas na própria amostra sob lupa. Quando a delimitação destes anéis apresentava um padrão morfológico diferente dos anéis destacadamente verdadeiros, os mesmos foram considerados falsos e a sua remoção foi efetivada após confirmação da melhoria da cronologia no programa Cofecha. De forma análoga, outros pesquisadores trabalhando com Cedrela spp. encontraram dificuldade na distinção dos anéis de crescimento, principalmente em função de delimitação difusa dos mesmos (Rauber, 2010; Lobão, 2011; Pereira, 2011).
O modelo de crescimento de Chapman-Richards (Equação 1) foi ajustado para todos os indivíduos, com exceção das árvores 10, 28 e 39, por apresentarem comportamentos distintos em relação às demais, e considerou apenas os primeiros 35 anos, em função da limitação dos dados a partir desta idade (Figura 3).

$d=71,918\left[1-0,951 \exp (-0,027 \text { Id })^{1,438}\right]$

Sendo que, 'd' é o diâmetro, variável dependente, e 'Id' é a idade, única entrada do modelo.

A determinação dos incrementos correntes anuais em diâmetro a partir da medição dos anéis de crescimento permitiu obter o incremento médio anual (IMA) em diâmetro, de $1,1 \mathrm{~cm}^{2} \mathrm{ano}^{-1}$, muito semelhante durante todo o período avaliado, oscilando entre 1,0 e 1,3 $\mathrm{cm}^{2} \mathrm{ano}^{-1}$. Esses valores estes compatíveis aos relatados por Iwasaki-Marochi (2007), que encontrou IMA em diâmetro variando de 0,68 a $1,04 \mathrm{a} \mathrm{cm} \mathrm{ano}^{-1}$. A diferença no ritmo de crescimento observada foi atribuída a diferentes níveis de competição nos primeiros anos do crescimento das árvores.

Em estudo desenvolvido em Floresta Ombrófila Mista na região centro sul paranaense com seis espécies nativas, entre elas Cedrela lilloi, dos quais foram coletados discos transversais à altura do peito, foi estimado, para os cinco indivíduos amostrados desta espécie, um IMA ${ }_{\text {DAP }}$ variando de 0,3 a 1,4 cm. As árvores avaliadas apresentavam idades entre de 16 e 122 anos. Os autores destacaram ainda que C. lilloi apresentou maior incremento periódico (IPA ${ }_{\mathrm{DAP} 10}=1,3 \mathrm{~cm}$ ) dentre as espécies avaliadas (Mattos et al., 2007).

Considerando o ritmo de crescimento observado no presente estudo, constou-se que um diâmetro alvo de $40 \mathrm{~cm}$ poderia ser alcançado entre 30 a 36 anos. Estes resultados indicam o grande potencial silvicultural da espécie no bioma Floresta Ombrófila Mista. De forma semelhante, Brienen \& Zuidema (2006) utilizaram as análises dos anéis de crescimento para o manejo de quatro espécies da Amazônia boliviana. Os crescimentos individuais para Cedrela odorata foram de 0,$4 ; 0,6 ; 1,0$ e $0,9 \mathrm{~cm}^{-1}$ para as classes diamétricas de 0 a $10 \mathrm{~cm}$, 10 a 30, 30 a 60 , acima de $60 \mathrm{~cm}$, respectivamente. Esses autores defendem o uso de tratos silviculturais para melhoria substancial das taxas de crescimento dos indivíduos suprimidos.

Boninsegna et al. (1989) avaliou 15 árvores de $C$. fissilis da Selva Missioneira na Argentina, domínio da Floresta Estacional Semi-Decidual, com séries cronológicas de até 200 anos. De acordo com as taxas 
de crescimento apresentadas pelos autores, observa-se que, a idade ótima de corte é de, aproximadamente, 60 anos de idade, quando as árvores apresentam cerca de $30 \mathrm{~cm}$ de diâmetro.

Comparando o ambiente de entorno das árvores amostradas observou-se que a árvore 10 estava, no momento da coleta, sob uma condição distinta das demais, compondo um sistema silvopastoril, com solo sujeito a pisoteio de animais. A interação desta atividade na compactação do solo, ao longo dos anos, pode ter exercido influência negativa sobre o crescimento desta árvore.

No caso da árvore 28 , verificou-se que a mesma se encontra às margens de uma estrada (Tabela 2) e que o ritmo de crescimento teve um aumento significativo a partir do $13^{\circ}$ ano de idade. Uma hipótese para este comportamento seria o fato de a estrada ter sido construída nessa época com a eliminação da vegetação próxima, acarretando disponibilização de maior espaço para o crescimento da copa e, consequentemente, maior incremento diamétrico.

Com relação à árvore 39 e considerando os argumentos discutidos por Iwasaki-Marochi (2007), pode-se inferir que, possivelmente, uma condição inicial de baixa competição permitiu o maior ritmo de crescimento inicial. Condição esta que não se manteve por toda a vida da árvore, pois a partir do $12^{\circ}$ ano de idade, houve redução considerável do ritmo de crescimento.

Outros fatores ambientais podem estar relacionados às variações no ritmo de crescimento de árvores ao longo da vida das mesmas, podendo ser temporários e de difícil detecção a posteriori. Segundo Pereira (2011), estudando árvores dominantes de cedro da Floresta Estacional Decidual na região de Santa Maria, RS, o crescimento diamétrico médio de árvores sem a presença de lianas $\left(1,6 \mathrm{~cm}^{2} \mathrm{ano}^{-1}\right)$ é significativamente superior ao crescimento de árvores com lianas $\left(0,8 \mathrm{~cm}^{2} \mathrm{ano}^{-1}\right)$.

As 28 séries de crescimento analisadas, totalizando 484 anéis anuais, com comprimento médio de 28 anos (1988-2009), apresentaram uma intercorrelação de 0,551 , superior ao valor crítico de 0,423 $(p<0,01)$, indicando existir correlação positiva e significativa entre as séries, e permitindo a construção de uma série dendrocronológica mestre para o período. Foi ajustado um modelo spline cúbico com rigidez relativa de $67 \%$.

A correlação de Pearson entre a maioria das variáveis meteorológicas e o crescimento radial não apresentaram resultados significativos, a exceção da umidade relativa (Figura 4). A ausência de correlação da maioria das variáveis pode ser explicada pela condição pluviométrica da área de estudo caracterizada por chuvas bem distribuídas durante todo o ano e ausência de períodos com déficit hídrico (Embrapa, 1988). Carvalho \& Stipp (2004), avaliando o balanço hídrico da região da Lapa, região do presente estudo, classificaram-no com ausência de déficit hídrico, com excedentes entre 798 e 1596 mm ano $^{-1}$.

Nos anos em que a precipitação ultrapassou 2.000 $\mathrm{mm}$, os indivíduos avaliados apresentaram crescimento indexado $<1$, indicando ritmo de crescimento abaixo do considerado normal para o período estudado.

Alguns trabalhos evidenciam baixa correlação do crescimento anual de espécies da Floresta Ombrófila Mista com as condições climáticas. Mattos et al. (2007) concluiu que o crescimento de cinco indivíduos de Cedrela lilloi não está significativamente correlacionado às condições ambientais locais. De forma semelhante, Pereira (2011) não encontrou correlação significativa entre temperatura média mensal e a série média de crescimento de $C$. fissilis. Porém, a autora constatou significância positiva do crescimento com a precipitação dos meses de junho, fevereiro e abril na região de Santa Maria, RS.

Oliveira et al. (2009), concluiu que o crescimento secundário de Araucaria angustifolia é determinado, principalmente, pelo comprimento do dia e temperatura, variáveis estas altamente associadas à evapotranspiração. Esse trabalho desenvolvido por Figueiredo Filho et al. (2003), com espécies nativas da Floresta Ombrófila Mista, os autores encontraram correlações do incremento diamétrico tanto com a temperatura como com a precipitação, esta, porém, de forma mais pronunciada.

Rauber (2010) codatou 12 indivíduos de C. fissilis em uma zona de ecótono de floresta subtropical Montana na região nordeste do Rio Grande do Sul e constatou que o crescimento é positivamente correlacionado à precipitação de dezembro a abril do ano anterior, como também, com a temperatura mínima dos meses de dezembro a março do ano anterior, no período de 1925 a 2004.

Em ambiente com sazonalidade climática de estação seca, Lobão (2011), estudando a fenologia de árvores de $C$. fissilis e $C$. odorata no Acre, verificou correlação significativa e positiva do crescimento com a precipitação do mês corrente e dos dois meses anteriores. Cabe destacar que se trata de ambiente totalmente diferente do presente estudo, principalmente com relação aos períodos seco e chuvoso. 

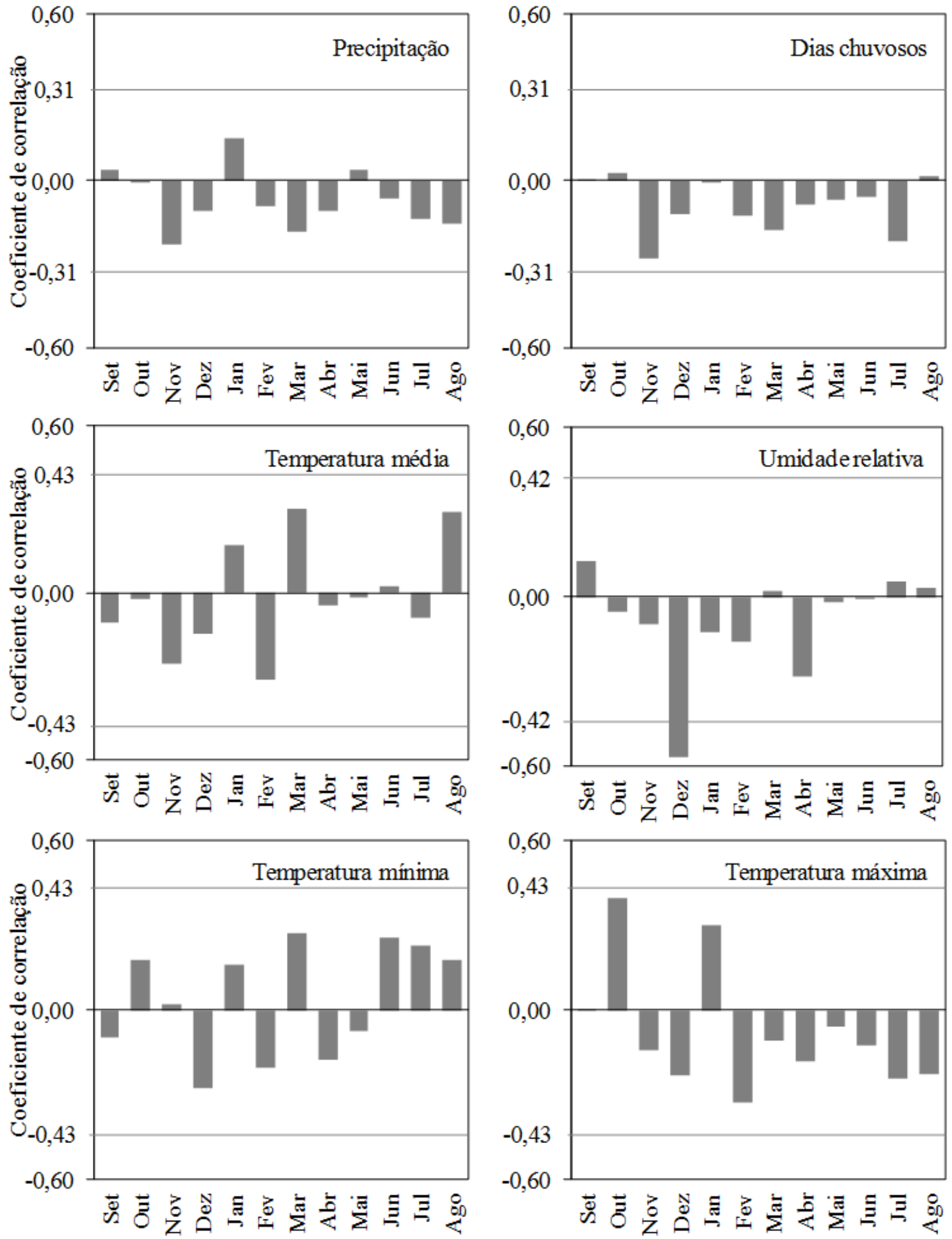

Figura 4. Coeficiente de correlação do crescimento radial das árvores de $C$. fissilis e precipitação, dias chuvosos, temperaturas média, mínima e máxima, e umidade relativa mensais. $\mathrm{O}$ valor crítico a partir do qual a correlação passa a ser significativa é $\pm 0,31$ para precipitação e dias chuvosos, $\pm 0,43$ para temperaturas média, mímina e máxima, e $\pm 0,42$ para umidade relativa. 
Em estudo desenvolvido em Aripuanã, MT, na região Amazônica, foram amostradas 30 árvores de Swietenia macrophylla e Cedrela odorata. Dünisch, O. et al. (2003), observaram atividade cambial durante todo o ano para $S$. macrophylla, enquanto que a $C$. odorata, esteve restrita ao período chuvoso de setembro do ano anterior a junho do ano corrente. Os autores encontraram correlação significativa entre a precipitação no final da estação de crescimento seguinte e a espessura do xilema nas regiões de incremento. $\mathrm{O}$ crescimento radial de Cedrela durante os dois primeiros meses depois do período de dormência foi atribuído à mobilização de recursos armazenados. Por fim, foram considerados estudos ecofisiológicos para explicar a maior amplitude ecológica do gênero Cedrela em relação ao Swietenia.

A umidade relativa em dezembro correlacionou inversa e significativamente com o crescimento radial (Figura 4). O resultado mais provável seria como obtido por Mattos et al. (2007) que relataram menor crescimento no mesmo ano ou no ano seguinte nos anos de 1999 e 2000, devido a reduzida precipitação e umidade relativa no início da primavera, e baixas temperaturas no inverno seguinte. $\mathrm{Ou}$ ainda, como Sette Junior et al. (2010) que atribuiram a redução do incremento em diâmetro do tronco nas árvores de Eucalyptus grandis, em janeiro de 2007, ao elevado teor de umidade do solo e ao maior número de dias nublados, afetando a respiração e translocação de nutrientes pelas raízes e fotossíntese.

A correlação de Pearson entre o crescimento da espécie e o número de geadas por ano não mostrou resultado significativo, apresentando o menor valor no inicio da estação de crescimento (setembro, $R=-0,29$ ) e o maior valor no final da estação de crescimento (agosto, $R=0,08$ ). Também foi possível observar que na maioria dos períodos em que o número de geadas foi inferior a dez, o índice de crescimento foi $>1$. A suscetibilidade de $C$. fissilis à geada é conhecida (Inoue, 1973; Carvalho, 1981; 1982), embora Inoue (1973), relate que plantios da espécie sob cobertura de Eucalyptus sp. não são afetados por geadas.

\section{Conclusões}

Para as condições estudadas, Cedrela fissilis apresenta incremento médio em diâmetro de $1,1 \mathrm{~cm}^{2} \mathrm{ano}^{-1}$. Visando um diâmetro de $40 \mathrm{~cm}$, a rotação proposta para a espécie é de 30 a 36 anos, indicando o potencial silvicultural da espécie na Floresta Ombrófila Mista.
Foi estabelecida uma série dendrocronológica significativa com intercorrelação de 0,551 para o período de 1988 a 2009 para a espécie na região.

A umidade relativa do ar em dezembro apresentou correlação inversa e significativa ao crescimento radial. As demais análises de correlação entre índice de crescimento de $C$. fissilis e as variáveis meteorológicas não apresentam valores significativos.

\section{Agradecimentos}

Os autores são gratos à Empresa Battistella Florestal e à equipe de pesquisa pelo apoio logístico e incentivo à pesquisa. À CAPES pelas bolsas de doutorado. ANA, EPAGRI, IAPAR e SIMEPAR pela cessão dos dados meteorológicos.

\section{Referências}

BONINSEGNA, J. A.; VILLALBA, R.; AMARILLA, L.; OCAMPO, J. Studies on tree rings, growth rates and age-size relationships of tropical tree species in Misiones, Argentina. IAWA Bulletin, Leiden, v. 10, p. $16-169,1989$.

BRIENEN, R. J. W.; ZUIDEMA, P. A. The use of tree rings in tropical Forest management: projecting timber yields of four Bolivian tree species. Forest Ecology and Management, v. 226, p. 256-267, 2006.

CARdoso, F. C. G.; MARQuES, R.; Botosso, P. C.; MARQUES, M. C. M. Stem growth and fenology of two tropical trees in contrasting soil conditions. Plant Soil, v. 354, p. 269-281, 2012.

CARVALHO, S. M.; STIPP, N. A. F. Contribuição ao estudo do balanço hídrico no Estado do Paraná: uma proposta de classificação qualitativa. Geografia, Londrina, v. 13, n. 1, p. 57-71, 2004.

CARVALHO, P. E. R. Competição entre espécies florestais nativas em Irati - PR, cinco anos após o plantio. Boletim de Pesquisa Florestal, Colombo, n. 2, p. 41-56, 1981.

CARVALHO, P. E. R. Comparação de espécies nativas, em plantio em linhas em capoeira, na região de Irati - PR, resultados aos sete anos. Boletim de Pesquisa Florestal, Colombo, n. 5, p. 53-68, 1982.

COPENHEAVER, C. A.; CRAWFORD, C. J.; FEARER, T. M. Agespecific responses to climate identified in the growth of Quercus alba. Trees, v. 25, n. 4, p. 647-653, 2011.

DOWNES, G. M.; WIMMWE, R.; EVANS, R. Understanding wood formation: gains to commercial forestry through tree-ring research. Dendrochronologia, v. 20, n. 1-2, p. 37-51 2002.

DÜNISCH, O.; MONTÓIA, V. R.; BAUCH, J. Dendroecological investigations on Swietenia macrphylla King and Cedrela odorata L. (Meliaceae) in the central Amazon. Trees, v. 17, p. 244-250, 2003.

EMBRAPA. Centro Nacional de Pesquisas Florestais. Zoneamento ecológico para plantios florestais no estado de Santa Catarina. Curitiba, 1988. 113 p. (EMBRAPA. CNPF. Documentos, 21). 
FIGUEIREDO FILHO, A.; HUBIE, S. do R.; SCHAAF, L. B.; FIGUEIREDO, D. J. de; SANQUETTA, C. R. Avaliação do incremento em diâmetro com o uso de cintas dendrométricas em algumas espécies de uma Floresta Ombrófila Mista localizada no Sul do Estado do Paraná. Revista Ciências Exatas e Naturais, v. 5, n. 1, 2003.

GRISSINO-MAYER, H. D. Ultimate tree-ring web pages. In: The science of tree rings. [Knoxville]: The University of Tennessee, 1996. Disponível em: < http://web.utk.edu/ grissino/ principles.htm\#2>. Acesso em: 30 jun 2013.

GRISSINO-MAYER, H. D. Computer assisted, independent observer, verification of tree-ring measurements. Tree Ring Bulletin, Tucson, v. 54, p. 29-41, 1997.

HOLMES, R. L. Dendrochronology program library. Tucson: Laboratory of Tree Ring Research, University of Arizona Tucson. 1994. Disponível em: <http://www.ltrr.arizona.edu/pub/dpl-mac/68k/ dpl.txt>. Acesso em: 17 jul. 2013.

HOLMES, R. L. The dendrochronology program library. In: GRISSINO-MAYER, H. D.; HOLMES, R. L.; FRITTS, H. C. International tree ring data dank: user's manual. Tucson: Laboratory of Tree Ring Research, University of Arizona, 1996.

IWASAKI-MAROCHI, C. Anéis anuais de crescimento do cedro (Cedrela fissilis - Meliaceae) aplicados à avaliação da taxa de crescimento e dendroclimatologia. 2007. $124 \mathrm{f}$. Tese (Doutorado em Engenharia Florestal) - Universidade Federal do Paraná, Curitiba.

INOUE, M. T. Ensaio de procedência de cedrela em Santo Antônio da Platina, PR. Revista Floresta, Curitiba, v. 4, n. 2, p. 49-57, 1973.

LOBÃO, M. S. Dendrocronologia, fenologia, atividade cambial e qualidade do lenho de árvores de Cedrela odorata L., Cedrela fissilis Vell. e Schixolobium parahyba var. amaxonicum Hub. Ex Ducke, no estado do Acre, Brasil. 2011. 215 f. Tese (Doutorado em Recursos Florestais) - Escola Superior de Agricultura "Luiz de Queiroz”, Universidade de São Paulo, Piracicaba.

MARCATI, C. R.; ANGYALOSSY, V.; EVERT, R. F. Seasonal variation in wood formation of Cedrela fissilis (MELIACEAE). IAWA Journal, Utrecht, v. 27, n. 2, p. 199-221, 2006.

MATTOS, P. P. de; SANTOS, A. T. dos; OliVEIRA, Y. M. M. de; ROSOT, M. A. D. Dendrocronologia de espécies da Floresta Ombrófila Mista do Município de Candói, PR. Pesquisa Florestal Brasileira, Colombo, n. 54, p. 153-156, jan./jun. 2007.

MATTOS, P. P. de; OLIVEIRA, M. F.; AGUSTINI, A. F.; BRAZ, E. M.; RIVERA, H. OLIVEIRA, Y. M. M. de; ROSOT, M. A. D.; GARRASTAZU, M. C. Aceleração do crescimento em diâmetro de espécies da Floresta Ombrófila Mista nos últimos 90 anos. Pesquisa Florestal Brasileira, Colombo, v. 30, n. 64, p. 319-326, 2010. DOI: 10.4336/2010.pfb.30.64.319

MEDEIROS, J. G. S.; TOMAZELLO FILHO, M.; KRUG, F. J.; VIVES, A. E. Tree ring characterization of Araucaria columnaris hook and its applicability as a lead indicator in environmental monitoring. Dendrochronologie, n. 26, p. 165-171, 2008.
MORELlATO, L. P. C.; TALORA, D. C.; TAKAHASI, A.; BENCKE, C. C.; ROMERA, E. C.; ZIPPARRO, V. B. Phenology of Atlantic Rain Forest trees: a comparative study. Biotropica, Washington, US, v. 32, n. 4, p. 811-823, 2000.

OLIVEIRA, B. R. U. de; LATORRACA, J. V. F.; TOMAZELLO FILHO, M.; GARCIA, R. A.; CARVALHO, A. M. de. Correlações dendroclimatológicas do Eucalyptus grandis Hill ex Maiden na região de Rio Claro, RJ. Ciência Florestal, Santa Maria, RS, v. 21, n. 3, p. 499-508, 2011.

OLIVEIRA, J. M.; SANTAROSA, E.; PILLAR, V. D.; ROIG, F. A. Seasonal cambium activity in the subtropical rain forest tree Araucaria angustifolia. Trees, n. 23, p. 107-115, 2009.

OLIVEIRA, J. M.; ROIG, F. A.; PILLAR, V. D. Climatic signals in tree-rings of Araucaria angustifolia in the Southern Brazilian highlands. Austral Ecology, Carlton, v. 35, n. 2, p. 134-147, 2010.

PEREIRA, L. D. Dendroecologia de Cedrela fissilis (Vell.) na Região de Santa Maria, RS. 2011. 66 f. Dissertação (Mestrado em Manejo Florestal) - Universidade Federal de Santa Maria, Santa Maria, RS.

PRETZSCH, H. Forest dynamics, growth and yield. London: Springer, 2009. $671 \mathrm{p}$.

RAUBER, R. C. Dendroecologia de Cedrela fissilis Vell. (Meliaceae) em um ecótono de Florestas Subtropicais Montanas no Brasil. 2010. 80 f. Dissertação (Mestrado em Ecologia) Universidade Federal do Rio Grande do Sul, Porto Alegre.

REITZ, R. Meliaceaes. Itajaí: Herbario Barbosa Rodrigues, 1984. (Flora Ilustrada Catarinense).

REITZ, R.; KLEIN, R. M.; REIS, A. Projeto madeira de Santa Catarina. Itajaí: Herbário Barbosa Rodrigues, 1978. 320 p.

RIZZINI, C. T. Plantas do Brasil: árvores e madeiras úteis do Brasil: manual de dendrologia brasileira. São Paulo: Edgard Blucher, 1978. 296 p.

SÃO PAULO (Governo do Estado). Secretaria do Meio Ambiente. Fundação Florestal. Florestar Estatístico. São Paulo, v. 11, n. 20, jun, 2008. Disponível em: < http://www.florestar.org.br/index. php?interna $=$ estatisticas/florestarestatistico $\&$ grupo $=3>$. Acesso em: 13 jan 2013.

SETTE JUNIOR, C. R.; TOMAZELLO FILHO, M.; DIAS, C. T. dos S.; LACLAU, J.P. Crescimento em diâmetro do tronco das árvores de Eucalyptus grandis W. Hill. Ex. Maiden e relação com as variáveis climáticas e fertilização mineral. Revista Árvore, Viçosa, MG, v. 34, n. 6, p. 979-990, 2010.

SEITZ, R. A. Utilidade da dendrocronologia na silvicultura. In: SIMPÓSIO BILATERAL BRASIL-FINLÂNDIA SOBRE ATUALIDADES FLORESTAIS, 1988, Curitiba. Anais... Curitiba: UFPR, 1988. p. 130-140.

SEITZ, R. A.; KANNINEN, M. Tree ring analysis of Araucaria angustifolia in Southern Brazil: preliminary results. IAWA Bulletin, Leiden, v. 10, p. 170-174, 1989. 
SCHWEINGRUBER, F. H. Wood structure and environment. Berlin: Springer, 2007. 279 p.

TOMAZELlO FILHO, M.; BOTOSSO, P. C.; LISI, C. S. Potencialidade da família Meliaceae para dendrocronologia em regiões tropicais e subtropicais. In: ROING, F. A. (Comp.). Dendrocronología em América Latina. EDIUNC: Mendoza, 2000. p. 381-431.
TOMAZELLO FILHO, M.; BOTOSSO, P. C.; LISI, C. S. Análise e aplicações dos anéis de crescimento das árvores como indicadores ambientais: dendrocronologia e dendroclimatologia. In: MAIA, N. B.; MARTOS, H. L.; BARELLA, W. (Org.). Indicadores ambientais: conceitos e aplicações. São Paulo: Educ, 2001. p. 117-143.

TOMAZELLO FILHO, M.; LISI, C. S.; HANSEN, N.; CURY, G. Anatomical features of increment zones in different species in the State of São Paulo, Brazil. Scientia Forestalis, Piracicaba, n. 66, p. $46-55,2004$. 
